\title{
THE EFFECT OF A CONTINUOUS INTRAVENOUS INFUSION OF INORGANIC PHOSPHATE ON THE RACHITIC LESIONS IN CYSTINOSIS
}

BY

\author{
ROBERT STEENDIJK \\ From the Department of Paediatrics, University of Amsterdam
}

(RECEIVED FOR PUBLICATION SEPTEMBER 19, 1960)

Fraser, Jaco, Yendt, Munn and Liu (1957) and Fraser, Geiger, Munn, Slater, Jahn and Liu (1958) reported healing of rachitic lesions in vitamin Ddeficient and in hypophosphataemic refractory rickets by intravenous administration of phosphate. The concentration of serum inorganic phosphorus, which initially had been very low, was kept at normal levels for a period of five to seven days. The authors concluded that vitamin $\mathrm{D}$ was not obligatory for healing in these types of rickets.

The metabolic defect in cystinosis leading to severe amino-aciduria, glycosuria, metabolic acidosis and deposition of cystine crystals in various organs, in addition to hypophosphataemia and rickets, is undoubtedly more severe than in infantile and primary refractory rickets. But there is no valid reason to suppose that the pathogenesis of rachitic lesions is different from that in other types of rickets, in which an insufficiency of calcium and a low concentration of serum inorganic phosphorus inhibit proper calcification of the bone matrix. However, this has never been proved. It was therefore decided to find out whether improved calcification could be obtained by inducing a lasting rise in the concentration of serum phosphorus in the absence of vitamin $D$ and alkalizing agents. Simultaneously, the effect of the induced rise in the concentration of serum phosphorus on the concentration of serum calcium and on the product of these concentrations was studied.

\section{Case Report}

The subject of this investigation was a 9-month-old boy, the fifth child of healthy parents who were first cousins. At the age of 6 months the first symptoms of the disease appeared (polyuria, polydipsia, failure to thrive); two months later he entered the Children's Hospital. On admission, physical examination revealed moderate malnourishment, craniotabes, rachitic rosary and thickening of the wrists. A slit-lamp examination of the cornea revealed the presence of numerous tiny crystals. Radiographs of the skeleton showed severe rickets.

Serum concentrations of calcium and inorganic phosphorus were 10.0 and $1.9 \mathrm{mg} . / 100 \mathrm{ml}$. respectively; the serum level of alkaline phosphatase was $17 \cdot 8$ Bodansky units. Plasma sodium, potassium and $\mathrm{CO}_{2}$ combining power were $137 \cdot 0,4.0$ and $13.5 \mathrm{mEq} /$ litre respectively. Results of liver function tests (thymol turbidity and cholesterol partition) were within normal limits. The creatinine clearance was $100 \mathrm{ml} . / \mathrm{min} . / 1 \cdot 73$ sq. $\mathrm{m}$. The specific gravity of the urine varied between 1,010 and 1,014; the 24-hour volume ranged from 600 to $800 \mathrm{ml}$. The urine contained a trace of albumen and 0.5 to $1 \%$ of reducing substances; the ratio amino-N to total $\mathrm{N}$ was $0 \cdot 19$.

\section{Methods}

Serum phosphorus was determined according to the method of Briggs (1922). Serum calcium was estimated colorimetrically by titration with ethylene diamino tetraacetate, using murexide as indicator.

For the infusion a sterile stock solution was prepared containing 1.8807 g. of $\mathrm{Na}_{2} \mathrm{HPO}_{4} 2$ aq. and $0.4502 \mathrm{~g}$. of $\mathrm{NaH}_{2} \mathrm{PO}_{4} 2$ aq. per $100 \mathrm{ml}$. of water. The $p \mathrm{H}$ of this solution was $7 \cdot 3$; it contained $416 \cdot 6 \mathrm{mg}$. of inorganic phosphorus per $100 \mathrm{ml}$. and it was isotonic with blood.

The total volume infused per 24 hours was $500 \mathrm{ml}$. It consisted of a mixture of $5 \%$ dextrose in water and the phosphate solution. The amount of phosphate infused initially was $750 \mathrm{mg}$. per 24 hours. Later it was derived by trial and error from the concentration of serum phosphorus, which was determined at least once every day. The aim was to obtain a serum level of at least $3.5 \mathrm{mg} . / 100 \mathrm{ml}$.

The fluid was administered by way of a polyethylene catheter which had been introduced in a saphenous vein. The rate of flow was kept constant throughout the time the infusion lasted.

\section{Results}

The effects of the infusion of inorganic phosphate on the serum levels of calcium and phosphorus are presented in the Table and Fig. 1. The induced 


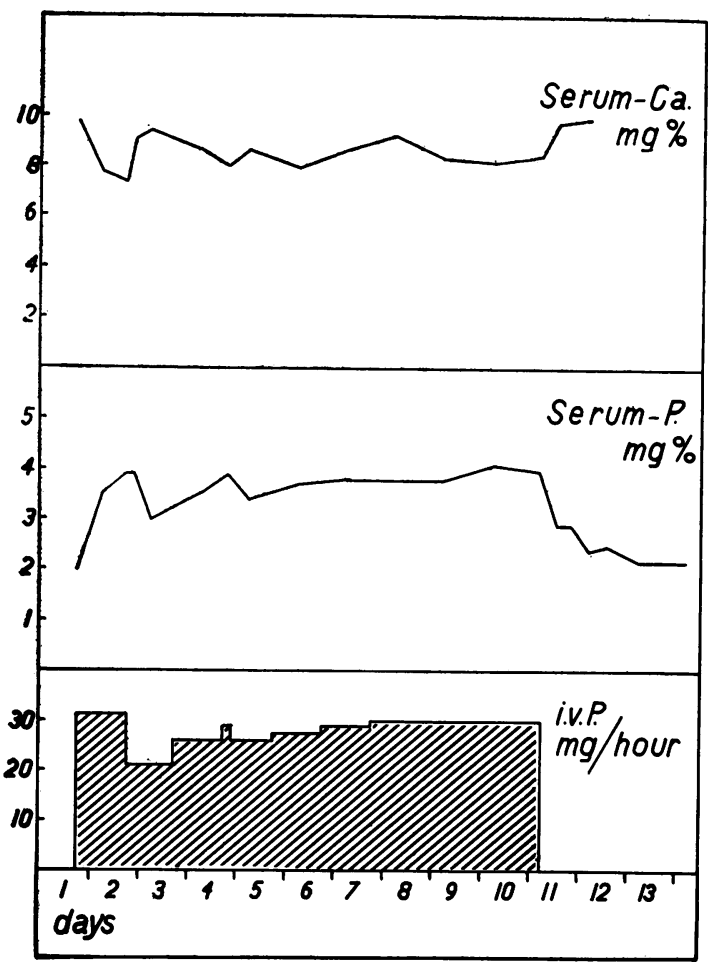

Fig. 1.-Relation between serum levels of calcium and phosphorus and rate of phosphate infusion.

TABLE

EFFECT OF CONTINUOUS INTRAVENOUS INFUSION OF PHOSPHATE ON SERUM LEVELS OF CALCIUM AND INORGANIC PHOSPHORUS

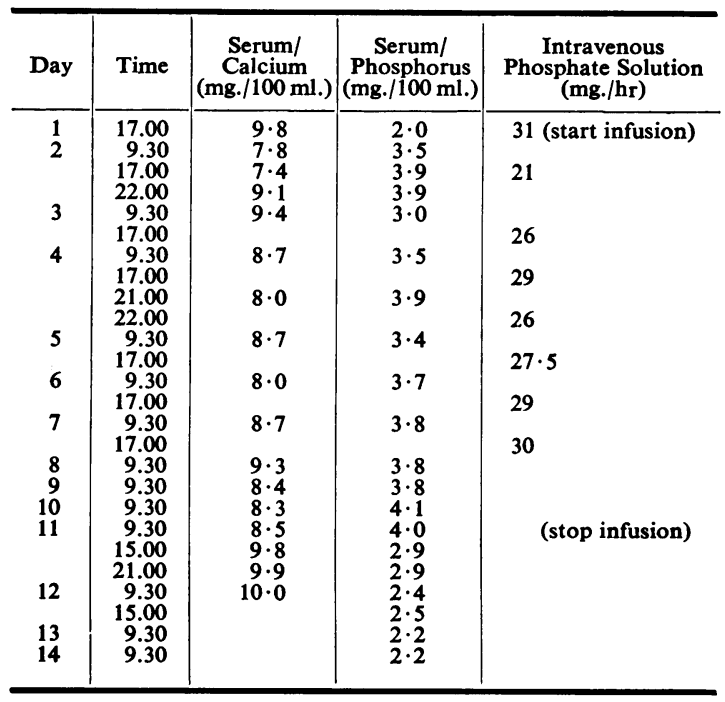

rise in serum phosphorus was accompanied by a corresponding fall in serum calcium. Subsequently, serum calcium rose slightly but did not attain its previous normal value until the infusion ceased. The infusion was continued for 10 days; two days later serum phosphorus reverted to its previous low value.

Radiographs of the right wrist were taken before the infusion, seven days after the beginning of the infusion, two days after the infusion had been discontinued and one week later. After seven days no signs of improved calcification were visible (Fig. 2a and b). Distinct lines of Mueller were present on the next radiograph however (Fig. 2c). After another week, during which no treatment was given, there was only negligible further deposition of bone salt (Fig. 2d).

Subsequent administration of vitamin D $(50,000$ I.U. per day) led to additional improvement, but complete healing was not achieved (Fig. $2 \mathrm{e}$ and $2 \mathrm{f}$ ).

The moderate acidosis and the intense aminoaciduria persisted during the administration of phosphate.

It can be concluded that the metabolic defect in cystinosis does not prevent increased deposition of bone salt at the rachitic metaphyses after intravenous administration of phosphate in the manner described and in the absence of vitamin $\mathrm{D}$.

\section{Discussion}

Hypocalcaemia. It is not easy to explain satisfactorily the hypocalcaemia which accompanied the induced rise in serum phosphorus. In normal individuals and in patients with primary hyperparathyroidism, given a large intravenous phosphate load, serum calcium shows a slight decrease which is corrected spontaneously within a very short time (Haak and Steendijk, 1960). After a large oral phosphate load serum calcium drops to very low levels in patients with vitamin $\mathrm{D}$ deficient rickets, but does not decrease in normal children (Jonxis, 1959). Apparently, the mechanism which maintains serum calcium at a normal level in the face of a rise in serum phosphorus is interfered with in rachitic conditions. Whether this should be ascribed to a diminished response of bone to endogenous parathyroid hormone (Jonxis, 1959), a different solubility of bone salt in rickets (Nicolaysen and Eeg-Larsen, 1956; Sobel, 1953), or a limited availability of calcium (Howard, 1953) is not known at present.

Source of Calcium Deposited. From the data obtained it cannot be concluded whether only a redistribution of skeletal calcium occurred, without 


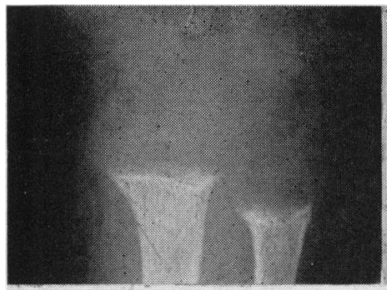

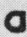

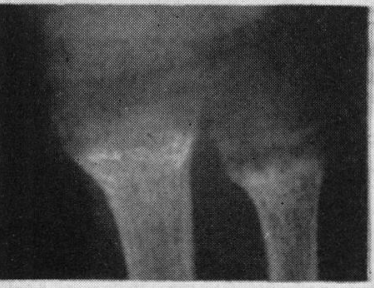

d

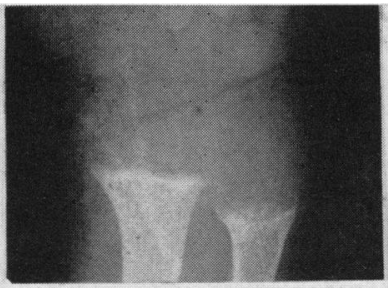

b

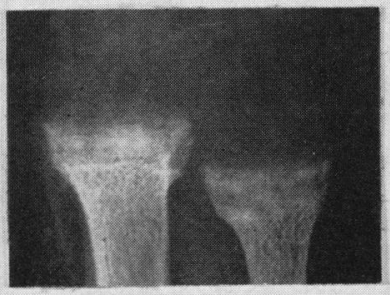

e

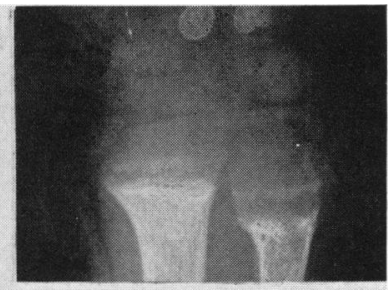

C

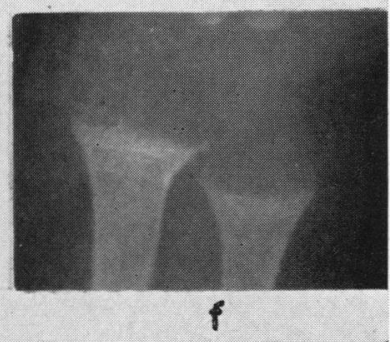

f

FIG. 2.-Radiographs of the right wrist.

(a) Before phosphate infusion.

(b) On seventh day of phosphate infusion.

(c) Two days after phosphate infusion.

change in retention, or, whether in addition, retention improved due to diminished urinary excretion or increased intestinal absorption of calcium.

Rate of Calcification. Approximately 40 years ago it was established empirically that the product of the concentrations of calcium and inorganic phosphate in serum $(\mathrm{Ca} \times \mathrm{P}$ product $)$ served as an index for the presence or absence of rickets. If the value of this product was too low, i.e. below 30 , it was thought that bone salt could not precipitate, thus leading to the development of rickets (Howland and Kramer, 1921). Recently it has been established that precipitation in the true sense of the word does not occur in bone. The mechanism by which the crystals are deposited is a different one, involving catalysed crystal nucleation, the precise nature of which is still a matter of dispute (Neuman and Neuman, 1958; Glimcher, 1959). All the same, it is now known that the rate of calcification (or the rate of nucleation and subsequent crystal growth) increases with an increasing ionactivity product of calcium and phosphate in the fluid bathing bone. At a fixed $p \mathrm{H}$ and ionic strength, the relationship between this product and the former $\mathrm{Ca} \times \mathrm{P}$ product is a linear one (McLean (d) 10 days after phosphate infusion.

(e) After three weeks of subsequent treatment with vitamin D.

(f) After nine weeks of subsequent treatment with vitamin D.

and Urist, 1955). Therefore the $\mathrm{Ca} \times \mathbf{P}$ product is a reliable index for the rate of calcification and has lost none of its previous usefulness.

Parenthetically it must be stated that the $\mathrm{Ca} \times \mathbf{P}$ product is not valid as an index for the rate of calcification in conditions of rickets associated with a severe glomerular insufficiency.

In the patient described the value of the $\mathrm{Ca} \times \mathbf{P}$ product before the infusion was $19 \cdot 6$, indicating a very slow rate of calcification and the presence of severe rickets. The average values for the concentrations of calcium and phosphorus during the infusion were 8.7 and 3.5 respectively; the average $\mathrm{Ca} \times \mathrm{P}$ product was 30.4 . In normal children of the same age this product is about 50 ; moderate rickets is present at a value of 30 . Even so, by a rise of the product from 20 to 30 , the rate of calcification was markedly increased, considering the fairly rapid appearance of lines of Mueller on the radiographs.

From experiments in vitro (Thomas, Connor and Howard, 1956; Sobel, Burger and Nobel, 1958), it was concluded that for initiation of calcification of rachitic cartilage a higher $\mathrm{Ca} \times \mathbf{P}$ product was necessary than for crystal growth once crystal nuclei had been deposited. Rachitic cartilage was found to show a considerable degree of calcification 


\title{
Ovarian metastases from primary gastrointestinal malignancies: the Royal Marsden Hospital experience and implications for adjuvant treatment
}

\author{
AE Taylor, VMC Nicolson and D Cunningham
}

The CRC Section of Medicine and the GI Unit, The Institute of Cancer Research and the Royal Marsden Hospital, Downs Road, Sutton, Surrey, UK.

\begin{abstract}
Summary We investigated the pattern and frequency of ovarian metastases in patients with primary gastrointestinal malignancies and evahuated the response to surgery, chemotherapy and in three cases radiotherapy. The literature reports that this group of patients have a poor prognosis, but no report has specifically addressed the response to chemotherapy. Using a database which is generated prospectively ${ }^{-}$we analysed 51 patients with primary gastrointestinal malignancies and ovarian metastases. All phtients received chemotherapy but only 36 were evaluable for response; five had adjuvant treatment and ten had nonmeasurable disease. Seventeen patients had surgical oophorectomy and three patients received radiotherapy. The overall response rate to chemotherapy was $22 \%$; eight partial responses and no complete stratified according to site of response, $11(31 \%)$ patients had a partial response at sites $=$ of extraovarian metastases and only five (14\%) had a partial response in the ovaries. Seven patients with piptmary colorectal cancer had a differential response in favour of extraovarian sites. The median survival was 9 months for the 51 patients. Three premenopausal women with resected gastric carcinoma received adjuvant chemotherapy and relapsed only in the ovaries. In primary colorectal tumours the response of ovariap metastases to chemotherapy is less than that for other sites. Therefore, the ovary may be a sanctuary sie for metastases which has important implications for adjuvant chemotherapy in women. These women coulP be followed up regularty by transvaginal ultrasonography to detect such metastases at an early stage wheto they would be amenable to surgical resection. Surgery should be considered for selected patients who develog metachronous metastases, as patients may be rendered disease free for several months.
\end{abstract}

Keyworke ovarian metastases; colorectal adjuvant chemotherapy

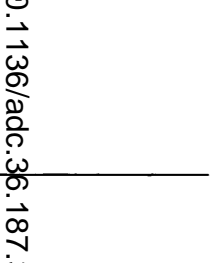

The ovary is a relatively frequent site of metastases from malignant neoplasms arising anywhere in the body. Ovarian metastases constitute $76 \%$ of genital tract metastases from extragenital primary tumours, of which $78 \%$ arise in the gastrointestinal tract (Mazur et al., 1984). Secondary tumours of the ovary constitute $10 \%$ of all ovarian neoplasms (Blaustein, 1982). In premenopausal women with colorectal cancer the incidence of ovarian metastases either found at the time of initial surgery or developing subsequently is reported to be between $13.2 \%$ and $25 \%$ (Recalde et al., 1974; Walton et al., 1976; MacKeigan and Ferguson, 1979). Similar rates have been reported for stomach cancer (Warren and Macomber, 1935; Webb et al., 1975). The term 'Krukenberg tumour' has become clinically synonymous with the presence of any metastasis to the ovaries, although purists contend that true Krukenberg tumours should meet the criteria established by Novak and Gray in 1938 and currently used by the WHO (Serov and Scully, 1973):

(1) presence of cancer in the ovary;

(2) intracellular mucin production by neoplastic signet-ring cells; and

(3) diffuse sarcomatoid proliferation of the ovarian stroma.

When lesions of this particular histology are analysed in terms of the primary neoplasm, gastric carcinoma is the most common source of ovarian metastases (Hale, 1968; Woodruff and Novak, 1960). Other ovarian metastases are of nonKrukenberg type and at times may be difficult to distinguish from primary ovarian carcinoma. The frequent use of computerised tomographic (CT) scanning nowadays to assess and follow up patients with tumours of the gastrointestinal tract results in the discovery of otherwise unsuspected ovarian metastases, the features of which are described in a separate report (in preparation). Many of the patients in this report did not have histological confirmation of malignancy in the ovary because they had never undergone oophorectomy.

Correspondence: D Cunningham

Received 3 March 1994; revised 28 July 1994; accepted 5 August 1994
However, the unequivocal change in ovainan size and appearance over the course of a patient's illngss was consistent with malignant involvement.

This report reviews ovarian metastases from primary gastrointestinal malignancies regardless of whether they were classical Krukenberg tumours or non-Krukeffberg tumours, as in many instances it was not possible $\overrightarrow{t 0}$ differentiate between the two. The pathological featiores of classical Krukenberg tumours are well described elsenibere (Wong et al., 1986). Classical Krukenberg tumourscape reported to occur in younger premenopausal women, occasionally occurring during or shortly after pregnancy (Ward, $\$ 966$; Holz and Hart, 1982), suggesting a hormonal effect $\frac{0}{2}$ one of the aetiological factors in its occurrence.

It has been postulated that the rearrangement of the ovarian surface in the post-ovulatory period and increased vascularity favour the seeding and growth for metastases (Sternberg, 1963). The diagnosis of Krukenberg tumours has been reported to be associated with a poor pregnosis (Mason and Kovalich, 1981), but the use of aggressivechemotherapy or surgery in these patients is not well described. Endocrinological symptoms related to ovarian dysfunction have been reported (Raquiz, 1991; Scully an 1961).

In this report we describe the clinical finding in patients with ovarian metastases in association with a primary gastrointestinal malignancy. Metastases were doctimented either surgically or by serial computerised tomography of the pelvis. All patients received chemotherapy at some stage of their illness, and we have assessed the response toutreatment. In addition, some patients underwent resection of their ovarian metastases, and three had pelvic radiotherapy. The potential benefits of these two therapeutic modalities Ifre discussed.

\section{Materials and methods}

\section{Patients}

Using a database which is generated prospecfively, we have analysed all patients presenting to the Royal Marsden Hos- 